\title{
Spice Model of a Piezo-Electric Transducer for Pulse- Echo System
}

\author{
Saleem Khan ${ }^{1}$, William Taube ${ }^{2}$, Nivasan Yogeswaran ${ }^{1,2}$, Hadi Heidari ${ }^{2}$, Ravinder Dahiya ${ }^{2}$ \\ ${ }^{1}$ Fondazione Bruno Kessler, Trento, 38123, Italy \\ ${ }^{1}$ Bendable Electronics and Sensors Technologies (BEST) Group, School of Engineering, University of Glasgow, G128QQ, UK \\ *Correspondence to: Ravinder.Dahiya@glasgow.ac.uk
}

\begin{abstract}
Pulse-echo system is the most widely used equipment in non-destructive testing (NDT). Herein we present the SPICE implementation of a piezo-electric transducer for pulseecho system. The SPICE model has been developed with an intention to obtain a virtual instrument for the pulse-echo system. The SPICE implementation of pulse-echo system includes the lossless piezoelectric transducer as an actuator and the sensor. The output of actuating stage is fed into the sensing stage of the sensor stage. The simulated results in the actuation and sensing modes are compared to the previously reported experimental results and the output trends are in good agreement. Findings from the comparative study, validate the application of our proposed model as a virtual system for pulse-echo system for non-destructive testing.
\end{abstract}

Keywords-Pulse Echo, Piezoelectric transducer, SPICE Modelling, Lossless Model, Sensors and Actuators.

\section{INTRODUCTION}

Nondestructive testing (NDT) are very attractive due to their capability of contactless evaluation of discontinuities in the surfaces and subsurface of materials or dissimilar mediums. This method has already secured a niche market for applications in various fields ranging from testing industrial materials to medical diagnosis and biomedical devices $[1,2]$. A range of NDT techniques for materials as well as continuous monitoring of mechanisms or supporting mediums have been developed. Few of the vastly used techniques for NDT are: time of flight diffraction (TOFD), digital X-ray imaging (DXR), 3D phase measurement, permanently installed monitoring sensors (PIMS), eddy current technology (ECT), high temperature hydrogen attack (HTHA) and pulse echo ultrasound testing [36]. Amongst these, pulse-echo method is preferred over other NDT methods due to its high penetration depth and accurate detection of surfaces/sub-surfaces flaws [2, 7, 8]. Furthermore, it is nonhazardous to operators and minimal sample preparation is required which provides instantaneous results. It is deployed on the single side of the specimen which is capable of producing detailed image and is highly accurate in determining reflector position and estimating size and shape of the flaw [9] .

The capabilities to measure discontinuities in the reflected waves in pulse-echo method, enables its validity in a range of applications. As a non-destructive testing technique, it is a very versatile technique and very often deployed in applications such as fragile materials testing, structural health monitoring, medical diagnosis and harsh environment testing where the device contact is limited or forbidden. It is widely used for materials testing for assessing flaw detection (cracks, inclusions, porosity, etc.), erosion \& corrosion thickness gauging, assessment of bond integrity in adhesively joined and brazed components, estimation of void content in composites and plastics, measurement of case hardening depth in steels and estimation of grain size in metals [2, 5-12]. At the same time, there has been an increasing demand of quality control for safety, especially in the aerospace, aircraft and automotive industry [13]. More importantly, the pulse-echo method forms the basis of many imaging methods in non-invasive medical diagnostics for humans and living organisms [14-17].

Designing and implementation of the pulse-echo testing system rely on the optimized performance of the transducer and accompanying electronic components. It is valuable to use some form of a theoretical model to assess, the performance of a transducer, the effects on the design changes, electronic modifications and varying parameters of the transducers [1, 1820]. Separate evaluation of the transducer and accompanying circuits may not provide the complete scope of the changing parameters, instead theoretical modelling and investigation of the combined system with transducer and electronics is more beneficial and can lead to an optimized evaluation of the performance $[18,21,22]$. For this reason, the circuit designing and simulating software like SPICE provides an attractive platform to investigate the model of pulse-echo method and different electronic circuits used in it. The capability to use SPICE to simulate the behavior of pulse-echo system in time and frequency domain makes it an attractive option. In this research, a lossless equivalent model of the piezoelectric transducer in Pulse Echo System is implemented in SPICE. Keeping the analysis simple, this study does not take into account the mechanical, electromechanical and dielectric losses. This paper is organized as follows: the pulse-echo method is presented in section II. Circuit design and SPICE simulations are presented in section III followed by results and discussion in section IV. The final section of the paper presents the conclusion and the future scope of this research.

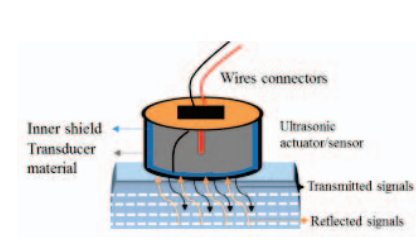

(a)

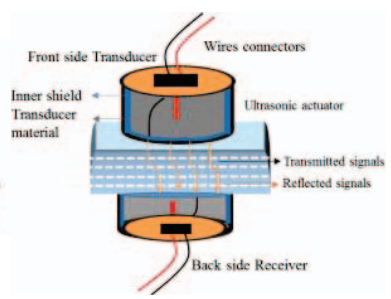

(b)
Fig. 1. Ultrasonic testing mechanisms based on transducer configuration, (a) Pulse-echo method having single side actuator/sensor (b). ThroughTransmission setup, transducer on both sides. Front side as an actuator and back side as a sensor/receiver. 


\section{PUlse ECHO MECHANISM}

Pulse-Echo method is an NDT, where beams of highfrequency sound waves are introduced into a material/medium under test for the detection of both surface and internal flaws [2, $7,16]$. The presence of defects causes changes/distortion in the received wave which is processed by the receiver circuitry to find out the defects' location and morphology. The key to the ultrasonic testing is the transducer which generates the ultrasonic waves. The same transducer can function as both actuator and receiver. The transducer can be configured either in a pulse echo configuration or a through transmission mode [7, 23-25]. Fig. 1 depicts the two different transducer configurations. In the case of pulse-echo test, the single transducer operates as both transmitter/actuator and receiver/sensor and it is placed on the only accessible side. Such types of transducers are usually developed from piezoelectric materials and have very promising values for electromechanical phenomena and vice-versa. In this research, we have developed a model for the simulation of transient behavior of piezoelectric transducer's actuation and sensing which can be further extended to a complete virtual system for simulating pulse-echo method and NDT.

\section{SPICE SiMULATIONS}

It would indeed be very cost-effective and time-effective to model a virtual testing system equivalent to a practical NDT testing setup where all the required parameters for the pulseecho system are investigated prior to their deployment in real system. A pulse-echo system, shown in the block diagram in Fig. 2 , usually consists of a transducer, sensor, pulser, receiver circuit, amplifier, signal conditioning and processing. Transducer is activated by pulses generated by pulse generator also called as firing circuit at optimal pulse frequency, width and delay. In this study, a lossless pulse-echo system has been implemented in PSPICE circuit simulator, commercially available from ORCAD. In our SPICE model we have implemented the transduction by considering the values of elastic dielectric, mechanical and piezoelectric parameters as shown in Fig. $3 b$ with electronic components, transmission line and Laplace functions. This work is an extension of the work done by one of the authors $[18,22]$. In the previous work, impedance characteristics of piezoelectric material in frequency domain has been investigated for various piezoelectric polymers. Two approaches have been done in this modeling. First approach is based on the transmission line model of the piezoelectric transducer. The sensor and the actuator comprises of the following components, namely: a static capacitor $\left(\mathrm{C}_{\text {polymer }}\right)$ to represent the piezoelectric material's capacitance, an electrical transmission line to represent the mechanical loss and "ELAPLACE" module to represent the electrical-mechanical conversion. The actuating mode component of the arrangement produces the force needed which travels as pressure waves, echoes and comes back as input to sensing component of the SPICE model. In the model in Fig. 3, the actuating stage's output as volts which signifies the force output is fed back as an input to the sensing stage which indicates the echo in the system. In a real pulse echo system there will be additional losses, delay and multiple echoes in the system which needs further detailed modelling. The second approach uses a Laplace equation to model the transient characteristics of the piezoelectric transducer, both while transmitting as well as receiving. The equation is given below

$$
\begin{aligned}
\frac{F(s)}{V(s)} \quad & H_{T X-P M}(s) \\
& =-h C_{o} \frac{Z_{F M}}{Z_{P M}+Z_{F M}}(1 \\
& \left.-\frac{Z_{P M}-Z_{B M}}{Z_{P M}+Z_{B M}} e^{-s T}\right)\left(1-e^{-s T}\right) \\
& /\left[\left(1-\left(\frac{Z_{P M}-Z_{B M}}{Z_{P M}+Z_{B M}}\right)\left(\frac{Z_{P M}-Z_{F M}}{Z_{P M}+Z_{F M}}\right) e^{-s T}\right)\right. \\
& -\left(\frac{k t^{2}}{2 T S}\right)\left(2 \frac{Z_{P M}}{Z_{P M}+Z_{F M}}(1\right. \\
& \left.-\left(\frac{Z_{P M}-Z_{F M}}{Z_{P M}+Z_{F M}}\right) e^{-s T}\right) \\
& +2 \frac{Z_{P M}}{Z_{P M}+Z_{B M}}(1 \\
& \left.\left.\left.-\left(\frac{Z_{P M}-Z_{B M}}{Z_{P M}+Z_{B M}}\right) e^{-s T}\right)\right)\left(-e^{-s T}\right)\right]
\end{aligned}
$$

Where $Z_{\mathrm{FM}}, \mathrm{Z}_{\mathrm{PM}}, \mathrm{Z}_{\mathrm{BM}}$ are the impedances of the front medium, piezoelectric medium and the back medium respectively. These were calculated considering three cases: heavy damping, medium damping and light tamping with a refection coefficients of $\mathrm{R}_{\mathrm{F}}=\mathrm{R}_{\mathrm{B}}=0.1, \mathrm{R}_{\mathrm{F}}=\mathrm{R}_{\mathrm{B}}=0.5, \mathrm{R}_{\mathrm{F}}=\mathrm{R}_{\mathrm{B}}=0.9$ respectively, where $\mathrm{R}_{\mathrm{X}}(\mathrm{X}=\mathrm{F}$ or $\mathrm{B}$ indicating front or back) is given by

$$
R_{X}=\frac{Z_{P M}-Z_{X M}}{Z_{P M}+Z_{X M}}
$$

The various material parameters for the modelling is taken from the experimental work by Hayward et al. [26] where a PZT $5 \mathrm{~A}$ piezoelectric transducer was used. $\mathrm{T}$ is the time delay for a

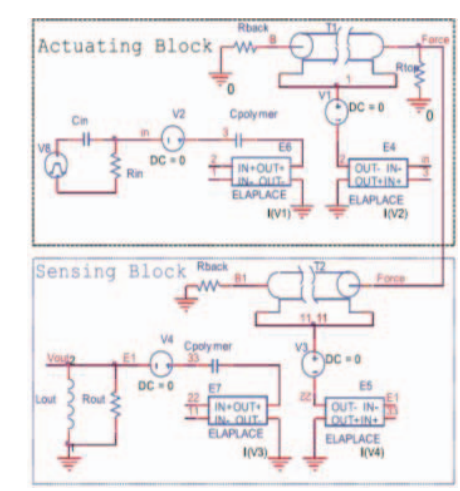

(a)

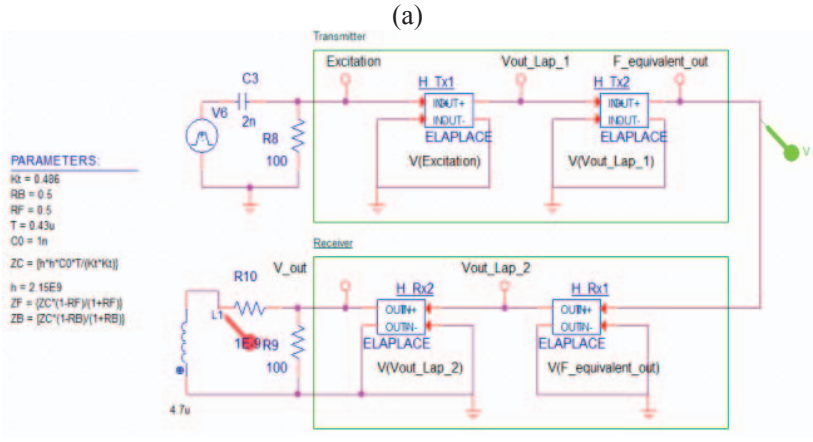

(b)

Fig. 3 Implementation of the SPICE model of piezoelectric polymer for standard Pulse-echo method (a) using transmission line method [18] (b) using direct Laplace equations 

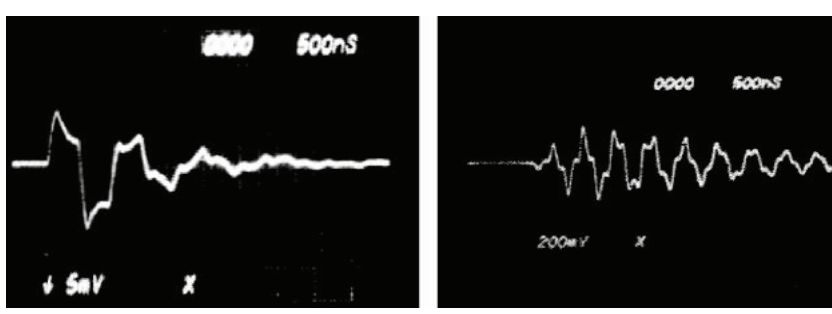

(a)
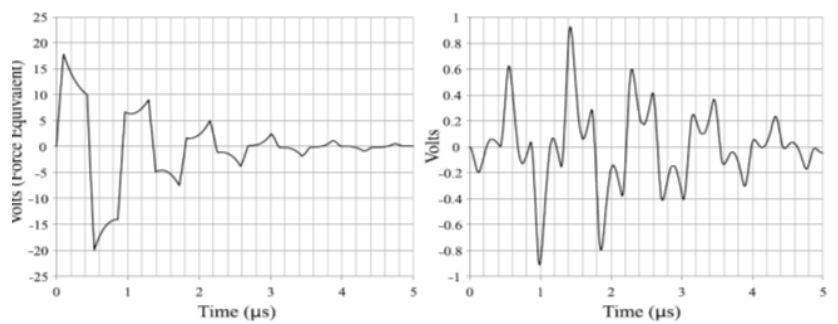

(b)

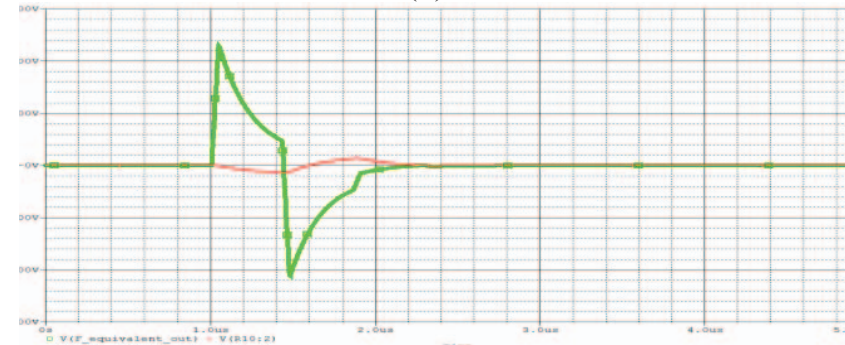

(c)

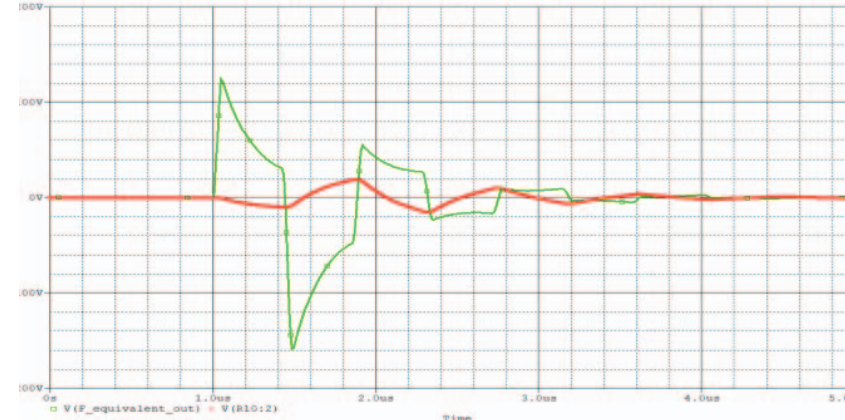

(d)

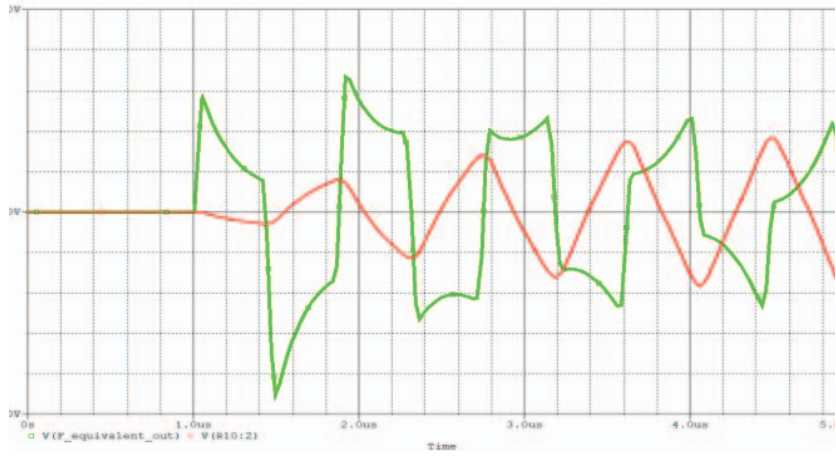

(e)

Fig. 4a (top-left) Experimental observation of force generated by piezoelectric material (actuating component) in the pulse-echo arrangement, reproduced from [26]; (top-right) Voltage output of piezoelectric material (sensing component) in the pulse-echo arrangement, reproduced from [26]; Simulated force equivalent voltage and output voltage obtained from SPICE model based on (b) transmission line method of piezoelectric material (actuating component) and direct laplace equations with (c) $\mathrm{RF}=\mathrm{RB}=0.1$ (Heavy damping) (e) $\mathrm{R}_{\mathrm{F}}=\mathrm{R}_{\mathrm{B}}=0.5$ (Medium damping) (f) $\mathrm{R}_{\mathrm{F}}=\mathrm{R}_{\mathrm{B}}=0.9$ (Low damping) wave to propagate through the Piezoelectric medium considered at $0.43 \mu \mathrm{s}$. $\mathrm{C} 0$ is the static capacitance (considered as $1 \mathrm{nF}$ ) of the piezoelectric transducer, $h$ is the piezoelectric charge coefficient $(\mathrm{h}=2.15 \mathrm{GV} / \mathrm{m})$ normally denoted by $\mathrm{h}_{33}$. The above is a modified equation of one reported by Hayward et al. [26] which relates the voltage across the transducer to the applied/generated force. In their study, they have used discrete Z-transform to simulate the transient behavior. The capability of SPICE simulation to perform the Laplace transform avoids the need of the complex Z-transform function reported in [26]. Furthermore the Z-transform equations has to be modified to incorporate the various firing/sensing circuits comprising of RL-C components in different configuration. In the Laplace equation approach, the 'ELAPLACE' option in SPICE is used which uses the convolution of impulse response with the input signal to get the transient output at various stages. Pulser/firing circuit components are directly introduced as lumped discrete component in the model which shows the simplicity of this approach compared to the Z-transform based approach. The input to the actuating stage of the both the SPICE model is a pulse of $300 \mathrm{~V}$ amplitude and with a fall time of $100 \mathrm{~ns}$ which is fed into a $\mathrm{RC}$ circuit consisting of $100 \Omega$ resistor $\left(\mathrm{R}_{\text {in }}\right)$ and $2 \mathrm{nF}$ capacitor $\left(C_{\text {in }}\right)$ as per the experimental setup in [26]. Also the output of the sensing stage is terminated with a load, comprising of a resistance $\left(R_{\text {out }}=100 \Omega\right)$ and inductance $\left(L_{\text {out }}=4.7 \mu \mathrm{H}\right)$ connected in parallel in accordance with the experimental setup in [26]. The netlist of the simulated SPICE model is provided in the Appendix.

\section{RESULTS AND DISCUSSION}

Fig. 4 shows that the simulated outputs of the model, both in sensing and actuating mode. The results presented in Fig. 4 show a comparison of the simulated results obtained by using the SPICE models to the experimental results reported in [26]. Fig. 4a left shows the experimentally observed trend of force measured on a PZT 5A piezoelectric transducer. The Fig. 4a right shows the voltage output of piezoelectric material acting as a sensor reproduced from [26]; Simulated force equivalent voltages and sensor output voltages obtained from SPICE model based on transmission line method of piezoelectric material is shown in Fig. $4 \mathrm{~b}$ and from direct Laplace equations are shown in Fig 4 (c) (d) and (e). where $\mathrm{R}_{\mathrm{F}}=\mathrm{R}_{\mathrm{B}}=0.1,0.5$ and 0.9 corresponding to Heavy damping, medium damping and low damping in their respective order. The medium damping result matches well with the experimental observation. The results clearly indicates that the simulated force equivalent voltage follows the same trend as that of experimentally observed result. The output characteristics of the transducer as sensor in the Laplace implementation differs slightly from the actual experimental characteristics. This is because in case of transmission line and the actual experiment sensing and actuation are coupled in time and the waves interact with each other. While in case of Laplace implementation, the output from the actuator is given as input to the sensor and the transmission/reflection during sensing is not coupled to the actuator. Such responses will be received by the sensor when the time between pulsing and receiving is higher than the actuators decay time. The overall simulated results of SPICE model are in good agreement with the experiment results, presented in [26].

\section{CONCLUSION}

A SPICE model of a piezoelectric transducer as a sensor and actuator for pulse-echo method is presented in this paper. A contact based pulse echo model is investigated without 
considering medium losses/effects where a single transducer is used both for actuation and sensing of the ultrasonic pulses. Further, at the input and output stages of the model, RLC circuit components of values based on an experimental setup has been used. Two approaches have been followed: one is based on transmission line model while the other uses simplified Laplace equation. The SPICE model was implemented and transient simulation of the model has been carried out in ORCAD PSPICE. The spice model is excited with a pulse input of specifications as per the experimental setup [30]. Simulation results from the SPICE model are compared with the experimental data. Comparative study shows a well matching trend between the simulated and experimental data, which validates our SPICE model. The model will be further optimized and extended into a virtual pulse echo system considering various losses, delay, medium effect and multiple echo arising in a real system.

\section{ACKNOWLEDGEMENT}

This work was supported by the European Commission under grant agreement PITN-GA-2012-317488-CONTEST and Engineering and Physical Science Council (EPSRC) Fellowship for Growth - Printable Tactile Skin (EP/M002527/1).

\section{REFERENCES}

[1] M. Yang and P. Qiao, "Modeling and experimental detection of damage in various materials using the pulse-echo method and piezoelectric sensors/actuators," Smart materials and structures, vol. 14, p. 1083, 2005.

[2] A. P. Mouritz, C. Townsend, and M. Z. Shah Khan, "Non-destructive detection of fatigue damage in thick composites by pulse-echo ultrasonics," Composites Science and Technology, vol. 60, pp. 23-32, 1/1/ 2000 .

[3] M. Willcox and G. Downes, "A brief description of NDT techniques," Insight NDT Equipment Limited, vol. 2003, 2000.

[4] R. D. Adams and P. Cawley, "A review of defect types and nondestructive testing techniques for composites and bonded joints," NDT international, vol. 21, pp. 208-222, 1988.

[5] A. G. Davis, F. Ansari, R. D. Gaynor, K. M. Lozen, T. J. Rowe, H. Caratin, et al., "Nondestructive Test Methods for Evaluation of Concrete in Structures," American Concrete Institute, ACI, vol. 228, 1998.

[6] J. García-Martín, J. Gómez-Gil, and E. Vázquez-Sánchez, "Nondestructive techniques based on eddy current testing," Sensors, vol. 11, pp. 2525-2565, 2011.

[7] G. Wróbel and S. Pawlak, "A comparison study of the pulseecho and through-transmission ultrasonics in glass/epoxy composites," Journal of Achievements in Materials and Manufacturing Engineering, vol. 22, pp. 51-54, 2007.

[8] P. Rizzo, A. Marzani, and J. Bruck, "Ultrasonic guided waves for nondestructive evaluation/structural health monitoring of trusses," Measurement Science and Technology, vol. 21, p. 045701, 2010.

[9] N. J. Carino, "The impact-echo method: an overview," in Proceedings of the 2001 Structures Congress \& Exposition, 2001, pp. 21-23.

[10] B. Zhao, Y. Jiang, O. A. Basir, and G. S. Mittal, "Foreign body detection in foods using the ultrasound pulse/echo method," Journal of food quality, vol. 27, pp. 274-288, 2004.

[11] B. A. Pellegrino and M. Nugent, "Nondestructive testing technologies and applications for detecting, sizing and monitoring corrosion/erosion damage in oil \& gas assets," NACE International Corrosion 2012, 2012.

[12] G. Marsh, "Finding flaws in composites," Reinforced Plastics, vol. 46, pp. 42-46, 2002.

[13] V. Giurgiutiu, Structural health monitoring: with piezoelectric wafer active sensors: Academic Press, 2007.

[14] P. Wells and N. T., Biomedical ultrasonics: Academic Pr, 1977.

[15] J. Karjalainen, "Novel Pulse-Echo Ultrasound Methods for Diagnostics of Osteoporosis," University of Eastern Finland. Department of Applied Physics. Dissertations in Forestry and Natural Sciences. Kuopio: Kopijyvä, 2011.
[16] J. J. Wild, "Application of echo-ranging techniques to the determination of structure of biological tissues," Classic Papers In Modern Diagnostic Radiology, p. 162, 2005.

[17] K. Nightingale, M. S. Soo, R. Nightingale, and G. Trahey, "Acoustic radiation force impulse imaging: in vivo demonstration of clinical feasibility," Ultrasound in medicine \& biology, vol. 28, pp. 227-235, 2002.

[18] R. S. Dahiya and M. Valle, Robotic tactile sensing: technologies and system: Springer Science \& Business Media, 2012.

[19] N. Aouzale, A. Chitnalah, and H. Jakjoud, "Experimental Validation of SPICE Modeling Diffraction Effects in a Pulse-Echo Ultrasonic System," Circuits and Systems II: Express Briefs, IEEE Transactions on, vol. 56, pp. 911-915, 2009.

[20] A. Puttmer, P. Hauptmann, R. Lucklum, O. Krause, and B. Henning, "SPICE model for lossy piezoceramic transducers," Ultrasonics, Ferroelectrics, and Frequency Control, IEEE Transactions on, vol. 44, pp. 60-66, 1997.

[21] E. Maione, P. Tortoli, G. Lypacewicz, A. Nowicki, and J. M. Reid, "PSpice modelling of ultrasound transducers: comparison of software models to experiment," Ultrasonics, Ferroelectrics, and Frequency Control, IEEE Transactions on, vol. 46, pp. 399-406, 1999.

[22] R. S. Dahiya, M. Valle, and L. Lorenzelli, "SPICE model for lossy piezoelectric polymers," Ultrasonics, Ferroelectrics, and Frequency Control, IEEE Transactions on, vol. 56, pp. 387-395, 2009.

[23] X. Yin, "The Study of Ultrasonic Pulse-echo Subwavelength Defect Detection Mechanism," University of Illinois at Urbana-Champaign, 2003.

[24] N. J. Carino, M. Sansalone, and N. N. Hsu, "A point source-point receiver, pulse-echo technique for flaw detection in concrete," in ACI Journal proceedings, 1986.

[25] A. L. Lopez-Sanchez and L. Schmerr, "Determination of an ultrasonic transducer's sensitivity and impedance in a pulse-echo setup," Ultrasonics, Ferroelectrics, and Frequency Control, IEEE Transactions on, vol. 53, pp. 2101-2112, 2006.

[26] G. Hayward and M. Jackson, "Discrete-time modeling of the thickness mode piezoelectric transducer," Sonics and Ultrasonics, IEEE Transactions on, vol. 31, pp. 137-150, 1984.

\section{APPENDIX}

Simulated SPICE Model's Netlist:

* source PIEZOTRANSIENT_BACK

E_H_Tx1 VOUT_LAP_10 LAPLACE $\{$ V(Excitation $)\}=\{(-\mathrm{h}) *(\mathrm{C} 0) *(2 *$

$\left.+\mathrm{ZF} /(2 *(\mathrm{ZC}+\mathrm{ZF}))) *\left(1-\mathrm{RF}^{*} \exp (-\mathrm{s} * \mathrm{~T})\right) *(1-\exp (-\mathrm{s} * \mathrm{~T})) * \exp (-\mathrm{s} * 2 \mathrm{n})\right\}$

E_H_Tx2 F_EQUIVALENT_OUT 0 LAPLACE $\{$ V(Vout_Lap_1) $\}=\{$

$+\quad 1 /\left(\left(1-\mathrm{RF}^{*} \mathrm{RB} * \exp \left(-\mathrm{s}^{*} 2 * \mathrm{~T}\right)\right)+((-\mathrm{Kt} * \mathrm{Kt} /(2 * \mathrm{~T})) *((2 * \mathrm{ZC} /(\mathrm{ZC}+\mathrm{ZF})) *((1-\right.$ $\left.\left.\left.\left.\left.\mathrm{RF}^{*} \exp (-\mathrm{s} * \mathrm{~T})\right)^{*}(1-\exp (-\mathrm{s} * \mathrm{~T})) / \mathrm{s}\right)\right)\right)\right)^{*} \exp (-\mathrm{s} * 4 \mathrm{n})$

+ \}

C_C3 PULSESOURCE EXCITATION 2n

R_R8 0 EXCITATION 100

V_V6 PULSESOURCE 0

+PULSE 3000 1u 0 100n

E_H_Rx1 VOUT_LAP_2 0 LAPLACE $\left\{\mathrm{V}\left(\mathrm{F} \_\right.\right.$equivalent_out $\left.)\right\}=\{$ (h)*(2/(ZC+

$\left.+\mathrm{ZF}))^{*}(1-(1+\mathrm{RB}) * \exp (-\mathrm{s} * \mathrm{~T})+\mathrm{RB} *(\exp (-2 * \mathrm{~s} * \mathrm{~T}))) * \exp (-\mathrm{s} * 2 \mathrm{n})\right\}$

E_H_Rx2 V_OUT 0 LAPLACE $\{$ V(Vout_Lap_2) $\}=\{$

$+1 /(\mathrm{s} *(1-\mathrm{RF} * \mathrm{RB} * \exp (-2 * \mathrm{~s} * \mathrm{~T})))\}$

R_R9 0 V_OUT 100

L_L1 0 N14462 $4.7 \mathrm{u}$

R R10 V OUT N14462 1E-9

.PARAM $\mathrm{c} 0=1 \mathrm{n} \mathrm{rb}=0.5 \mathrm{~h}=2.15 \mathrm{e} 9 \mathrm{rf}=0.5 \mathrm{kt}=0.486 \mathrm{zb}=\{\mathrm{zc} *(1-\mathrm{rb}) /(1+\mathrm{rb})\} \mathrm{zc}=$ $+\{\mathrm{h} * \mathrm{~h} * \mathrm{c} 0 * \mathrm{t} /(\mathrm{kt} * \mathrm{kt})\} \mathrm{zf}=\{\mathrm{zc} *(1-\mathrm{rf}) /(1+\mathrm{rf})\} \mathrm{t}=0.43 \mathrm{u}$ 